\section{ORIGINAL RESEARCH}

B.S. Choi

J.H. Kim

C. Jung

J.-M. Hwang

\title{
High-Resolution 3D MR Imaging of the Trochlear Nerve
}

BACKGROUND AND PURPOSE: The cisternal segment of the trochlear nerve is difficult to identify reliably by routine MR imaging. We investigated the visibility and anatomic features of the trochlear nerve by using high-resolution 3D-bTFE imaging in healthy subjects.

MATERIALS AND METHODS: This study was conducted with 32 healthy subjects without ocular movement disorders. For us to visualize the cisternal segment of the trochlear nerve, all subjects underwent 3D-bTFE imaging at 3T with 2 different resolutions: conventional resolution (voxel size, $0.67 \times 0.45 \times 1.4 \mathrm{~mm}$ ) and high resolution (voxel size, $0.3 \times 0.3 \times 0.25 \mathrm{~mm}$ ). Visibility of the trochlear nerve was graded with the use of a qualitative scale of certainty as follows: definite, probable, and indeterminate. The diameter of the trochlear nerve was measured.

RESULTS: On conventional-resolution images, the visibility of the trochlear nerve was definite in 3 nerves, probable in 12 nerves, and indeterminate in 49 nerves. On high-resolution images, visibility was definite in 63 nerves and probable in 1 nerve. The mean diameter of the trochlear nerve was 0.54 $\mathrm{mm}$ (range, $0.35-0.96 \mathrm{~mm}$ ).

CONCLUSIONS: The trochlear nerve was visualized $100 \%$ of the time on high-resolution imaging with a voxel smaller than the nerve diameter. High-resolution imaging should have an important role in investigating the pathogenic mechanism of neuropathic strabismus, such as congenital superior oblique palsy.

ABBREVIATIONS: 3D-bTFE $=3 \mathrm{D}$ balanced turbo-field echo; $\mathrm{NA}=$ not applicable; $\mathrm{SENSE}=$ sensitivity-encoding; SNR = signal-to-noise ratio

$\mathbf{T}$ rochlear nerve palsy is the most frequent isolated cranial neuropathy that affects ocular motility. ${ }^{1}$ The etiologic mechanism of the disease is unclear. Congenital aplasia or hypoplasia of the oculomotor or abducens nerve has been documented on MR imaging in patients with congenital oculomotor nerve palsy and Duane retraction syndrome. ${ }^{2,3}$ However, the presence or absence of the trochlear nerve has not been investigated in congenital superior oblique palsy, which is the most common type of trochlear nerve palsy. Recently, several MR imaging studies have been conducted to identify the trochlear nerve ${ }^{4-8}$; however, these studies did not consistently demonstrate it due to its small size, even in healthy subjects.

In this study, we used MR imaging with much higher resolution compared with previous studies in which the voxel size was smaller than the diameter of the trochlear nerve. The visibility and anatomic features of the trochlear nerve by using this sequence were investigated in healthy subjects and compared with conventional sequences.

Received September 25, 2009; accepted after revision November 11.

From the Departments of Radiology (B.S.C., J.H.K., C.J.) and Ophthalmology (J.-M.H.), Seoul National University College of Medicine, Seoul National University Bundang Hospital, Seongnam, Korea.

This study was supported by grant 11-209-023 from the Research Fund of Seoul National University Bundang Hospital.

Please address correspondence to Jae Hyoung Kim, MD, Department of Radiology, Seoul National University Bundang Hospital, 300 Gumi-dong, Bundang-gu, Seongnam-si, Gyeonggi-do 463-707, Korea; e-mail: jaehkim@snu.ac.kr

DOl 10.3174/ajnr.A1992

\section{Materials and Methods}

\section{Subjects}

This study was conducted with 34 healthy subjects (18 males and 16 females; age range, 3-59 years; mean age, 39 years) without any ocular movement disorder. Informed consent was obtained from all subjects, and our institutional review board approved the study protocol.

\section{MR Imaging Sequences}

MR imaging was conducted by using a 3T system (Intera Achieva; Philips Medical Systems, Best, the Netherlands) with a SENSE head coil. All subjects underwent 3D-bTFE imaging with 2 different resolutions: conventional and high resolution. Conventional-resolution imaging was performed in an axial plane, including the midbrain, pons, and upper medulla oblongata. The sequence parameters were as follows: TR, $6.2 \mathrm{~ms}$; TE, $3.1 \mathrm{~ms}$; flip angle, $60^{\circ}$; FOV, $150 \times 150 \mathrm{~mm}$; matrix, $224 \times 333$; section thickness, $1.4 \mathrm{~mm}(0.7-\mathrm{mm}$ overlap with the adjacent section); voxel size, $0.67 \times 0.45 \times 1.4 \mathrm{~mm}$; 70 sections; SENSE factor, 1.5; acquisition time, 3 minutes 39 seconds.

High-resolution imaging was performed at the lower midbrain and upper pons, including the inferior margin of the inferior colliculus, which is known as the level of the root exit zone of the trochlear nerve. The scanning plane was set to an oblique axial direction perpendicular to the long axis of the aqueduct, which was approximately parallel to the course of the trochlear nerve. The sequence parameters were as follows: TR, $9.9 \mathrm{~ms}$; TE, $5.0 \mathrm{~ms}$; flip angle, $60^{\circ}$; FOV, $150 \times$ $150 \mathrm{~mm}$; matrix, $500 \times 500$; section thickness, $0.25 \mathrm{~mm}$; voxel size, $0.3 \times 0.3 \times 0.25 \mathrm{~mm}$; 60 sections; SENSE factor, 2; acquisition time, 7 minutes 14 seconds.

\section{Image Analysis}

Two neuroradiologists analyzed the MR images and determined the grade of visibility of the trochlear nerve by consensus. Datasets were 
Visibility of 64 trochlear nerves on conventional- and highresolution 3D-bTFE imaging

\begin{tabular}{|c|c|c|c|}
\hline \multirow[b]{2}{*}{ Visibility Grade } & \multicolumn{2}{|c|}{ Conventional } & \multirow{2}{*}{$\begin{array}{c}\text { High-Resolution } \\
\text { No. of } \\
\text { Cases }(\%)\end{array}$} \\
\hline & $\begin{array}{c}\text { No. of } \\
\text { Cases (\%) }\end{array}$ & $\begin{array}{c}\text { False-Positive } \\
\text { Cases }(\%)\end{array}$ & \\
\hline Definite & $3(4.7)$ & $1 / 3(33.3)$ & $63(98.4)$ \\
\hline Probable & 12 (18.3) & $3 / 12(25)$ & $1(1.6)$ \\
\hline Indeterminate & $49(76.6)$ & NA & 0 \\
\hline
\end{tabular}

anonymized and presented to the examiners in a random order. The conventional-resolution images were interpreted first; then the highresolution images were interpreted at another session 2 weeks later to minimize recall bias.

Visibility of the trochlear nerve was graded with the use of a qualitative scale of certainty, with a grading of definite, probable, and indeterminate. The criteria of "definite" required both of the following: 1) a curvilinear nonbranching structure in the perimesencephalic cistern coursing in the anterolateral direction toward the ipsilateral tentorium, and 2) identification of the root exit point at the posterior aspect of the pontomesencephalic junction (ie, at the level of the inferior margin of the inferior colliculus). If the first requirement was fulfilled but the second requirement was not fulfilled, the grade was given as "probable." If both requirements were not fulfilled, the grade was given as "indeterminate." In several cases, images were reformatted in various oblique planes to trace the course of the trochlear nerve. Visibility was examined statistically by using the $\chi^{2}$ test.

To validate the results of conventional-resolution images, we anatomically correlated the trochlear nerves with definite or probable visibility as determined on conventional-resolution images with the findings on reference standards, which were high-resolution images in this study.

To evaluate the size of the trochlear nerve, we measured the diameter on high-resolution images in all subjects. Measurements were performed 3 times and averaged.

\section{Results}

Of 34 subjects, 2 subjects were excluded from the study due to the degradation of imaging quality by severe motion artifacts. Ultimately, 64 nerves of 32 subjects were analyzed.

Visibility of the trochlear nerve was significantly different between the conventional- and high-resolution images (Table). On conventional-resolution images, visibility was definite in $4.7 \%$ (3 of 64 nerves), probable in 18.8\% (12 of 64 nerves), and indeterminate in $76.6 \%$ ( 49 of 64 nerves) of cases. On high-resolution images, visibility was definite in $98.4 \%$ (63 of 64 nerves) and probable in 1.6\% (1 of 64 nerves) of cases. Visibility was significantly different between the 2 sequences $(P<.0001)$. When considering "probable" as positive identification of the trochlear nerve, the nerve was identified in $100 \%$ (64 of 64 nerves) of cases on high-resolution images and was identified in $23.4 \%$ (15 of 64 nerves) of cases on conventional-resolution images. Visibility was significantly different between the 2 sequences $(P<.0001)$.

Of 15 trochlear nerves with grades of definite or probable visibility on conventional-resolution images, 11 nerves were concordant with the findings of high-resolution images (Table) (Figs 1 and 2). However, 4 nerves were found to be falsepositive; the nerves were proved to be the perimesencephalic vessels by high-resolution imaging (Figs 3 and 4). The mean diameter of the trochlear nerve was $0.54 \mathrm{~mm}$ (range, 0.35$0.96 \mathrm{~mm})$.

\section{Discussion}

The aim of this study was to compare high-resolution 3DbTFE imaging with conventional imaging in visualizing the trochlear nerve in healthy subjects on a $3 \mathrm{~T}$ MR imaging system. This technique provides a T2 over T1 contrast and can be very useful in regions with tissues with very different $\mathrm{T} 2$ relaxation times, such as CSF and neural structures. ${ }^{9}$ Thus, 3DbTFE imaging can depict the small cranial nerves with high tissue contrast and high spatial resolution, as in previous MR imaging studies. ${ }^{4,5,7,10}$

The identification of the trochlear nerve on MR imaging is related to the spatial resolution of the imaging, anatomic configuration (ie, the size and course) of the nerve, and proximity of the nerve to adjacent vessels with a similar course and caliber. Recently, several MR imaging studies have been conducted to visualize the trochlear nerve in healthy subjects ${ }^{4,5,7}$; however, the nerve has not been consistently identified. Cheng et $\mathrm{al}^{4}$ used a $0.35 \times 0.35 \times 1.0 \mathrm{~mm}$ voxel (size of the $\mathrm{x}$-axis, $y$-axis, and z-axis) and reported definite identification of the trochlear nerve in $16 \%$ of cases, probable identification in $22 \%$ of cases, and no identification in $62 \%$ of cases. Fischbach et $\mathrm{al}^{5}$ used a $0.37 \times 0.47 \times 2.0 \mathrm{~mm}$ voxel at a field strength of $3 \mathrm{~T}$ and reported $85 \%$ detectability, including blurred as well as distinct visualization. Yousry et $\mathrm{al}^{7}$ used a $0.35 \times 0.69 \times 0.69 \mathrm{~mm}$ voxel and visualized the trochlear nerve definitely in $75 \%$ of cases and probably in $20 \%$ of cases, the best level of detection ever reported. We think that the resolution of the $\mathrm{z}$-axis (ie, section thickness) used in the previous studies was too large to identify the trochlear nerve consistently. The mean diameter of the trochlear nerve was only $0.54 \mathrm{~mm}$ (range, 0.35-0.96 $\mathrm{mm}$ ) in our study, which is similar to the diameter reported in a previous cadaver study (mean, $0.85 \mathrm{~mm}$; range, $0.5-1.1$ $\mathrm{mm}) .{ }^{11}$ In addition to its small size, the trochlear nerve courses horizontally in the perimesencephalic cistern, approximately parallel to the imaging plane. Therefore, the higher $\mathrm{z}$-axis resolution used in our protocol compared with previous studies must have been a key factor in increasing the identification rate of the trochlear nerve. We used a $0.3 \times 0.3 \times 0.25 \mathrm{~mm}$ voxel for which the resolution of the $\mathrm{z}$-axis was smaller than the nerve diameter for $3 \mathrm{~T}$ imaging, and the nerve was visualized in $100 \%$ of cases (definite, $98.4 \%$; probable, $1.6 \%$ ).

Another obstacle to identifying the nerve is its proximity to the perimesencephalic vessels with a similar course and caliber. These vessels include the superior cerebellar artery and its branches, and the brachial tributaries of the precentral cerebellar vein. ${ }^{12}$ Villain et al ${ }^{13}$ reported that $90 \%$ of the trochlear nerves had $\geq 1$ contact with the superior cerebellar artery or its branches. To discriminate the trochlear nerve from these vessels, Yousry et $\mathrm{al}^{7}$ additionally performed gadolinium-enhanced 3D time-of-flight MR angiography. In our study, much higher resolution imaging could trace the entire course of the trochlear nerve from its exit point and was able to discriminate the nerve from adjacent vessels in all subjects without the need for MR angiography. Oblique reformation of the images also provided easy tracing of the nerve in several cases. 

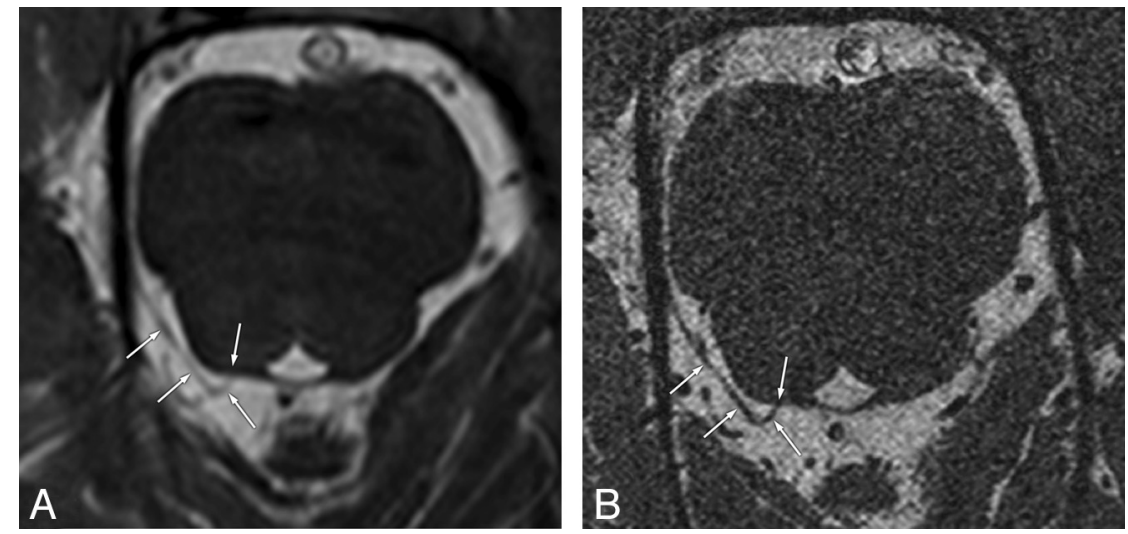

Fig 1. Concordant visibility of the trochlear nerves on conventional- and high-resolution 3D-bTFE images in a 48-year-old man. Conventional- $(A)$ and high-resolution $(B)$ images show clearly the cisternal segment of the right trochlear nerve (arrows) with definite visibility from the root exit point at the posterior aspect of the pontomesencephalic junction to the ipsilateral tentorium.
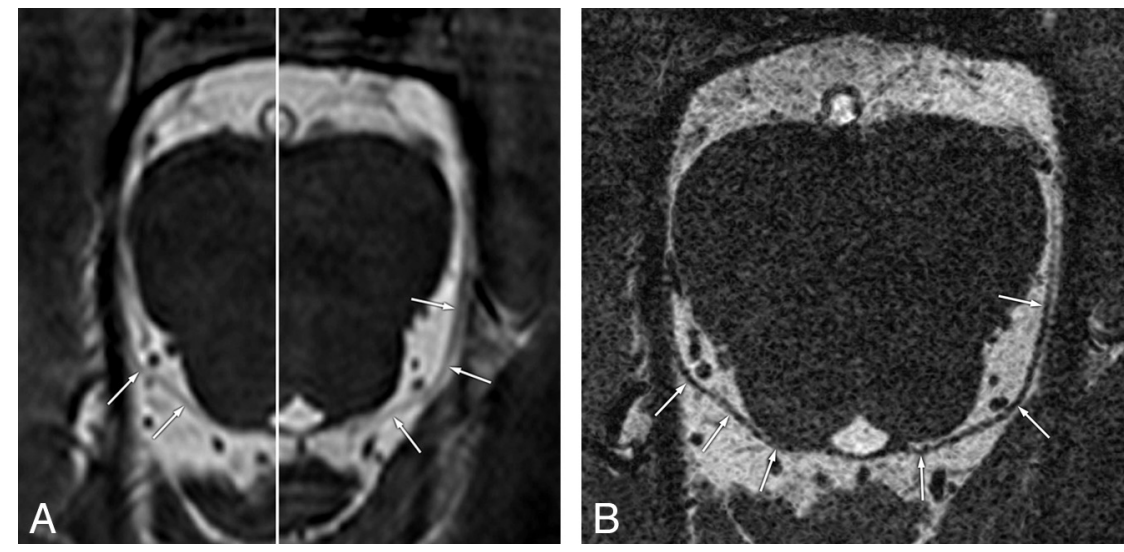

Fig 2. Concordant visibility of the trochlear nerves on conventional- and high-resolution 3D-bTFE images in a 34-year-old man. A, Conventional image with a fusion of different right and left levels shows curvilinear nonbranching structures (arrows) with "probable" visibility, coursing in an anterolateral direction toward the ipsilateral tentorium. $B$, Reformatted high-resolution image clearly shows the trochlear nerves bilaterally (arrows) from the root exit points with "definite" visibility.
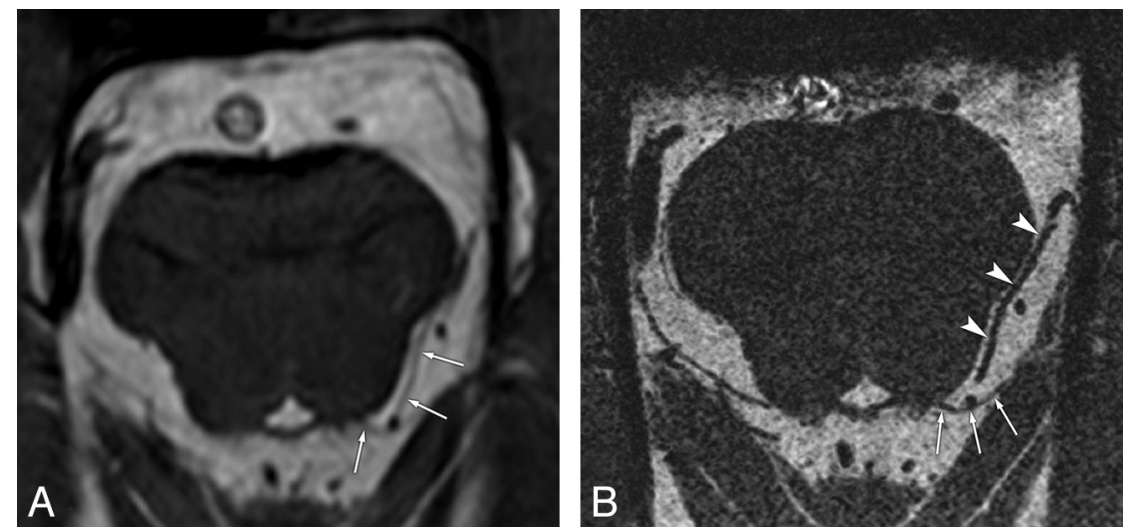

Fig 3. False-positive case of the trochlear nerve on a conventional-resolution 3D-bTFE image in a 47-year-old man. A, Conventional image shows the root exit point and its cisternal course of the left trochlear nerve (arrows) with "definite" visibility. B, Reformatted high-resolution image clearly separates the left trochlear nerve (arrows) from the vessel (arrowheads), which is misinterpreted as the trochlear nerve in $A$.

There are several limitations to our study. First, highresolution 3D-bTFE imaging has a relatively poor SNR and a long scanning time. The SNR is linearly proportional to the voxel volume ${ }^{14}$; therefore, the small voxel has a negative effect on the SNR. ${ }^{15}$ In addition, a long scanning time due to high spatial resolution may exacerbate motion artifacts on images. With continuing development of coil and parallel imaging technology, this limitation should be over- come to a great extent. Second, only 3 pediatric control subjects were included in the study. Superior oblique palsy is the most common pediatric ocular motility disorder. ${ }^{1} \mathrm{~A}$ narrower cisternal space and smaller trochlear nerve in children than in adults may perturb consistent identification of the trochlear nerve. Therefore, a further study including a substantial number of pediatric control subjects is needed. 

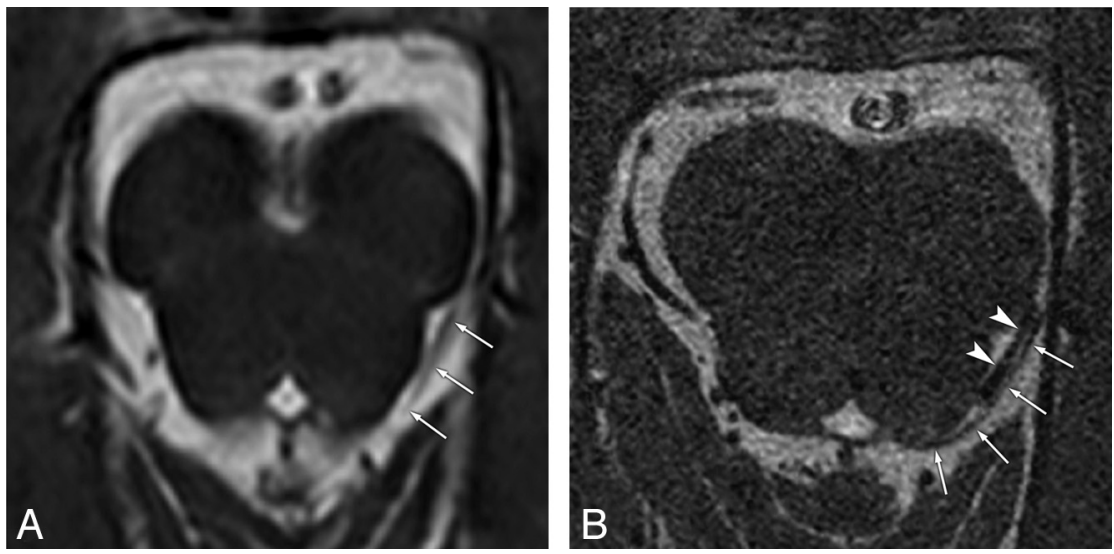

Fig 4. False-positive case of the trochlear nerve on conventional-resolution 3D-bTFE image in a 3-year-old girl. $A$, Conventional image shows the cisternal segment of the left trochlear nerve (arrows) with "probable" visibility. B, Reformatted high-resolution image clearly separates the left trochlear nerve (arrows) from the adjacent vessel (arrowheads) running parallel, which is misinterpreted as the trochlear nerve in $A$.

\section{Conclusions}

The trochlear nerve can be consistently visualized on highresolution imaging with a voxel smaller than the nerve diameter. High-resolution imaging should have an important role in investigating abnormalities of the trochlear nerve.

\section{References}

1. Holmes JM, Mutyala S, Maus TL, et al. Pediatric third, fourth, and sixth nerve palsies: a population-based study. Am J Ophthalmol 1999;127:388-92

2. Kim JH, Hwang JM. Magnetic resonance imaging in three patients with congenital oculomotor nerve palsy. Br J Ophthalmol 2009;93:1266-67

3. Kim JH, Hwang JM. Usefulness of MR imaging in children without characteristic clinical findings of Duane's retraction syndrome. AJNR Am J Neuroradiol 2005;26:702-05

4. Cheng YS, Zhou ZR, Peng WJ, et al. Three-dimensional-fast imaging employing steady-state acquisition and T2-weighted fast spin-echo magnetic resonance sequences on visualization of cranial nerves III - XII. Chin Med J (Engl) 2008;121:276-79

5. Fischbach F, Müller M, Bruhn H. High-resolution depiction of the cranial nerves in the posterior fossa (N III-N XII) with 2D fast spin echo and 3D gradient echo sequences at 3.0 T. Clin Imaging 2009;33:169-74
6. Yousry I, Camelio S, Schmid UD, et al. Visualization of cranial nerves I-XII value of $3 \mathrm{D}$ CISS and T2-weighted FSE sequences. Eur Radiol 2000;10:1061-67

7. Yousry I, Moriggl B, Dieterich M, et al. MR anatomy of the proximal cisternal segment of the trochlear nerve: neurovascular relationships and landmarks. Radiology 2002;223:31-38

8. Solsberg M, Fournier D, Potts D. MR imaging of the excised human brainstem a correlative neuroanatomic study. AJNR Am J Neuroradiol 1990;11:1003-13

9. Scheffler K, Lehnhardt S. Principles and applications of balanced SSFP techniques. Eur Radiol 2003;13:2409-18

10. Demer JL, Ortube MC, Engle EC, et al. High-resolution magnetic resonance imaging demonstrates abnormalities of motor nerves and extraocular muscles in patients with neuropathic strabismus. J AAPOS 2006;10:135-42

11. Ettl A, Salomonowitz E. Visualization of the oculomotor cranial nerves by magnetic resonance imaging. Strabismus 2004;12:85-96

12. Tubbs RS, Oakes WJ. Relationships of the cisternal segment of the trochlear nerve. J Neurosurg 1998;89:1015-19

13. Villain M, Segnarbieux F, Bonnel F, et al. The trochlear nerve: anatomy by microdissection. Surg Radiol Anat 1993;15:169-73

14. Bushberg JT, Seibert JA, Leidholdt EM, et al. The Essential Physics of Medical Imaging. Baltimore: Lippincott Williams \& Wilkins; 1994

15. Hendrick RE. Image contrast and noise. In: Stark DD, Bradley WG Jr, eds. Magnetic Resonance Imaging. 3rd ed. St Louis: Mosby; 1999:43-67 